\title{
Qualidade de vida de mulheres negras com câncer de mama: uma revisão de literatura
}

\author{
Esthér Aparecida Silva dos Santos* \\ Tereza Cristina Cavalcanti Ferreira de Araujo**
}

\begin{abstract}
Resumo
Há mais de duas décadas, a Organização da Mundial da Saúde propôs uma definição para Qualidade de Vida (QV) e, desde então, numerosas pesquisas têm sido desenvolvidas em diferentes especialidades. Caracterizada pela multidimensionalidade, subjetividade e dinamismo, a QV abrange diversos domínios da existência humana, incluindo-se fatores de natureza sociocultural, como gênero e etnia. Considerando a necessidade de ampliar conhecimentos específicos sobre QV, realizou-se uma revisão da literatura especializada, publicada entre 2014 e 2019, sobre QV de mulheres negras com câncer de mama. Para tanto, foram consultadas as bases BVS Psi, SciELO, MEDLINE/PubMed. Identificaram-se oito artigos, cuja análise apontou alguns indicadores relacionados à percepção negativa da QV: sintomas depressivos e ansiosos, idade inferior a 50 anos, estar em quimioterapia e tipo de câncer. Constatou-se, também, que bem-estar espiritual/religioso sobressaiu como fator associado a uma percepção de QV mais favorável. Concluiu-se que mais estudos são necessários, notadamente para fundamentar intervenções em Oncologia.
\end{abstract}

Palavras-chave: qualidade de vida, câncer da mama, origem étnica e saúde

\section{Quality of Life of Black Women with Breast Cancer: A Literature Review}

\begin{abstract}
More than two decades ago, the World Health Organization proposed a definition for Quality of Life (QOL) and, since then, numerous studies have been developed in different specialties. Characterized by multidimensionality, subjectivity and dynamism, QOL comprises several domains of human existence, including factors of a socio-cultural nature, such as gender and ethnicity. Considering the need to expand specific knowledge about QOL, a review of the literature published between 2014 and 2019 on the QOL of black women with breast cancer, was carried out. The BVS Psi, SciELO, MEDLINE/PubMed databases were consulted. Eight articles were identified, whose analysis pointed out some indicators related to the negative perception of QOL: depressive and anxious symptoms, age below 50 years, being on chemotherapy and type of cancer. It was also found that spiritual / religious well-being stood out as a factor associated with a more favorable perception. It was concluded that more studies were needed.
\end{abstract}

Keywords: quality of life, breast neoplasms, ethnicity and health

* Graduanda em Psicologia na Universidade de Brasília (UnB).

** Doutorado em Psychologie pela Université de Paris X - Nanterre e Profa. Titular da UnB. 


\section{ESTHÉR APARECIDA SILVA DOS SANTOS \\ TEREZA CRISTINA CAVALCANTI FERREIRA DE ARAUJO}

\section{Introdução}

Inicialmente, cabe relembrar que há mais de duas décadas a Organização Mundial de Saúde (OMS) propôs uma definição bastante ampla de Qualidade de Vida (QV): "a percepção do indivíduo de sua posição na vida, no contexto de sua cultura e no sistema de valores em que vive e em relação a suas expectativas, seus padrões e suas preocupações" (Fleck, 2008, p. 25). Desde então, inúmeros projetos de pesquisa foram efetivados no intuito de gerar conhecimentos teóricos e práticos sobre o tema. Até mesmo revistas especializadas foram criadas para difusão dos trabalhos - por exemplo Applied Research in Quality of Life, Quality of Life Research e Health and Quality of Life Outcomes - além de sites e plataformas que, periodicamente, atualizam dados e ferramentas de medida (Matos \& Araujo, 2015). Todavia, essa profusão de informações veiculadas em diferentes campos do conhecimento acarreta, em algumas ocasiões, um uso abusivo do termo e sua vulgarização. De fato, muitas outras noções - como bem-estar, satisfação, felicidade e saúde - atravessam o campo conceitual da QV, tal como apontado pela literatura (Costa Neto \& Araujo, 2008; Fleck, 2008; Matos \& Araujo, 2015).

Vale esclarecer que um exame atento desse 'constructo' permite evidenciar ao menos três atributos constitutivos da QV. Primeiramente, sua multidimensionalidade alude às várias dimensões da existência humana - tais como, os domínios físico, social, psicológico e ambiental - as quais devem ser ponderadas para se alcançar uma concepção suficientemente abrangente. A maioria dos autores também entende que a QV é intrinsecamente subjetiva, pois ela se caracteriza pela percepção da própria pessoa acerca da sua experiência vital. Além disso, em razão das transformações inerentes ao desenvolvimento humano, a QV denota-se pelo seu dinamismo (Costa Neto \& Araujo, 2008; Matos \& Araujo, 2015).

Nomeadamente em saúde, muitos pesquisadores e profissionais optaram por empregar o termo 'qualidade de vida relacionada à saúde’ e, ao longo do tempo, numerosas medidas gerais e específicas têm sido propostas desde que, em 1948, foi produzida a Perfomance Status Scale de Karnofsky e Burchenal, destinada a enfermos oncológicos. Em virtude da complexidade das vivências de saúde, múltiplos domínios são avaliados em estudos de QV nessa área: funcionamento locomotor, alimentação, sono, relações familiares, interações sociais, atividade laboral, sexualidade, espiritualidade e aspectos psicológicos (Costa Neto \& Araujo, 2008; Yin, Njai, Barker, Siegel, $\&$ Liao, 2016).

\section{Câncer, Gênero e Etnia}

À título introdutório, é pertinente destacar que ao se pensar 'saúde', é obrigatório reconhecer a determinação de fatores socioculturais, tais como gênero e etnia, os quais influenciam fortemente as experiências de pacientes, seus familiares e comunidade (Gurung, 2010). Assim, Bergerot, Araujo e Tróccoli (2014) investigaram interrelações de câncer e gênero, comparando o nível de distress de homens e mulheres acometidos por um câncer. A amostra foi constituída por 328 pacientes com idade entre 18 e 86 anos, portadores de diferentes tipos de câncer. Para avaliação do distress - definido como o 'sexto sinal vital' pela American Cancer Society -, foi aplicada a ferramenta Termômetro de Distress (TD) em três etapas do tratamento quimioterápico: início, meio e fim. Os autores verificaram maiores taxas de distress entre as mulheres, as quais também reportaram maior número de dificuldades nos domínios 'físico', 'emocional', 'prático', 'familiar' e 'espiritual'. Em contraposição, os homens revelaram ter mais problemas práticos e físicos. Abreviadamente, Bergerot et al. (2014) corroboraram a literatura especializada, pois reafirmaram particularidades da experiência oncológica vivenciada pelos gêneros masculino e feminino.

Em especial no que se refere ao câncer de mama, é importante ressaltar que essa enfermidade resulta do crescimento desordenado de células invasivas, que ocorre a partir de alterações genéticas, as quais podem ser tanto adquiridas quanto hereditárias (Instituto Nacional de Câncer, 2018). Trata-se do tipo de câncer de maior incidência em mulheres no mundo $(24,2 \%)$, sendo a principal causa de morte por câncer em mulheres (15\%) (World Health Organization, 2018).

No Brasil, estimativas do Instituto Nacional do Câncer (2019) apontaram 59.700 casos de câncer de mama em 2019, o que representaria 29,5\% da incidência de cânceres em mulheres. No tocante às diferenças étnicas, as taxas parecem similares em mulheres negras e brancas. Porém, o índice de mortalidade devido à doença é maior entre mulheres negras (Silva, Araújo, \& Câmara, 2017). Sob mesma ótica, Nogueira, Guerra, 
Cintra, Corrêa, Fayer e Bustamante-Teixeira (2018) notaram disparidade étnica nos índices de sobrevida ao câncer de mama. Dez anos após a doença, apenas 44\% das mulheres negras encontravam-se recuperadas, em contraste com 69,5\% de mulheres brancas sobreviventes de um câncer. Além disso, as mulheres negras tiveram um pior prognóstico clínico, bem como apresentaram um estadiamento mais avançado do tumor no momento do diagnóstico. Outrossim, Renna Júnior e Silva (2018) constataram que mulheres pardas e mulheres negras tendem a iniciar o tratamento oncológico mais tardiamente em comparação com mulheres brancas.

No cenário brasileiro, o reconhecimento da diversidade étnica-cultural foi fortalecido pela instituição da Política Nacional de Saúde Integral da População Negra (PNSIPN) e por orientações gerais voltadas para os profissionais de saúde no sentido de ofertar cuidados centrados nas necessidades desse segmento populacional (Ministério da Saúde, 2013). Mas, ainda persistem desigualdades no acesso aos serviços de saúde, sobretudo de pessoas de baixa renda, com menor nível de escolaridade e de etnias 'não brancas' (Vieira, Formenton, \& Bertolini, 2017). Inclusive, López (2012) alertou quanto ao: 'funcionamento cotidiano de instituições e organizações, que operam de forma diferenciada na distribuição de serviços, benefícios e oportunidades aos diferentes segmentos da população do ponto de vista racial" (p. 127). Em outras palavras, estereótipos étnicos, somados a estereótipos de gênero perturbam a relação usuário-profissional de saúde, comprometendo o alcance das metas terapêuticas traçadas pela equipe especializada em cuidados oncológicos.

Considerando, portanto, os interesses assistenciais e científicos envolvidos na ampliação dos conhecimentos sobre gênero e etnia em Oncologia, empreendeu-se uma revisão da literatura especializada sobre qualidade de vida de mulheres negras com câncer de mama.

\section{Método}

Convém evocar que uma revisão sistemática da literatura especializada visa identificar, selecionar e analisar os dados disponíveis sobre um determinado assunto (Galvão \& Pereira, 2014). No presente trabalho, foram consultadas as seguintes bases: Biblioteca Virtual em Saúde - Psicologia (BVS Psi), Biblioteca Eletrônica Científica Online (SciELO) e Sistema Online de Busca e Análise de Literatura Médica (MEDLINE/PubMed). A busca foi feita por meio de termos escolhidos no vocabulário Descritores em Ciências da Saúde (DeCS) - idealizado pelo Centro Latino-Americano e do Caribe de Informação em Ciências da Saúde (BIREME) -, a saber: "qualidade de vida" (quality of life), "mulher negra" (black woman) e "câncer de mama" (breast cancer).

Assim sendo, estipularam-se quatro critérios para seleção dos artigos divulgados nessas bases: (1) estudos sobre QV em mulheres negras com câncer de mama; (2) estudos em língua portuguesa, inglesa ou espanhola; (3) estudos disponíveis integralmente em alguma das bases consultadas; e (4) estudos publicados entre 2014 e 2019.

\section{Resultados e Discussão}

$\mathrm{Na}$ BVS PSI, não foram identificados trabalhos com os três descritores associados. Na SciELO, também não se encontrou artigo indexado por meio dos termos "qualidade de vida", "mulher negra" e "câncer de mama", sendo que a busca apenas com o descritor "qualidade de vida" apontou a disponibilidade de 90 artigos. Tendo em vista que ambas as bases são massivamente adotadas para difusão e acesso aos periódicos brasileiros, tais constatações revelam a insuficiência da produção nacional sobre o assunto.

No que tange às bases interrelacionadas MEDLINE/ PubMed, foi possível levantar 157 artigos, quando não foram aplicados os critérios previamente definidos para a presente pesquisa: texto integralmente gratuito e publicação de 2014 a 2019. É oportuno assinalar, também, que ao se empregar quality of life, breast cancer e, tão somente, woman, foram informados 5.706 artigos. Após a inclusão do termo black woman (e mantendo-se os demais critérios de busca), identificaram-se 51 artigos, o que revela disparidade entre a produção científica sobre os dois temas, inclusive no âmbito internacional. Ou seja, nota-se pouca produção que focalize uma dimensão étnica em relação à QV de mulheres acometidas por um câncer de mama.

A leitura dos resumos resultou na seleção de oito artigos que atenderam a todos os parâmetros estabelecidos. A Tabela 1 apresenta tais trabalhos, os quais serão analisados e discutidos a seguir. 


\section{ESTHÉR APARECIDA SILVA DOS SANTOS \\ TEREZA CRISTINA CAVALCANTI FERREIRA DE ARAUJO}

Tabela 1 - Artigos sobre QV de mulheres negras com câncer de mama $(\mathrm{N}=8$

Título

Racial differences in quality of life and employment outcomes in insured women with breast cancer

Effect of age and race on quality of life in young breast cancer survivors

The effects of symptoms on quality of life during chemotherapy in african-american women with breast cancer

Understanding racial differences in health-related quality of life in a population-based cohort of breast cancer survivors

To be young, black, and living with breast cancer: a systematic review of health-related quality of life in young black breast cancer survivors

Examining health-related quality of life patterns in Pinheiro et al. (2017) women with breast cancer

Health-related quality of life in black breast cancer survivors with and without triple-negative breast cancer (TNBC)

Health-related quality of life of african-american female breast cancer survivors, survivors of other cancers, and those without cancer
Autores e Ano de Publicação

Bradley \& Wilk (2014)

Morrow et al. (2014)

Gaston-Johansson et al. (2015)

Pinheiro et al. (2016)

Samuel et al. (2016)
Face à escassez de literatura observada nesse levantamento, é preciso retomar resumidamente os artigos extraídos. Assim, Bradley e Wilk (2014) estudaram uma amostra de 548 mulheres, sendo que 22\% eram afro-americanas. As autoras examinaram as respostas ao Questionário Genérico de Avaliação de Qualidade de Vida (SF-36) e à Center for Epidemiological Scale Depression (CES-D) em três momentos diferentes (etapa diagnóstica, aos dois e nove meses após o início do tratamento), bem como dados sobre emprego no intervalo de 2007 a 2011. As mulheres negras obtiveram menor pontuação na dimensão física do SF-36 nos três períodos. Também manifestaram maior nível de sintomas depressivos antes mesmo de iniciarem a quimioterapia. Em contrapartida, no período de dois e nove meses após o começo do tratamento, as mulheres afro-americanas tiveram escores mais favoráveis na escala de saúde mental e depressão, em comparação com as mulheres brancas. É possível cogitar que as mulheres negras, a medida em que confrontavam as adversidades do tratamento, foram compelidas a desenvolver estratégias adaptativas. Essas constatações, igualmente, permitem pensar que nos momentos iniciais do acompanhamento clínico, é necessário dar mais atenção ao surgimento de sintomas depressivos e ansiosos que podem dificultar a adesão terapêutica.

No que diz respeito especificamente à questão laboral, a pesquisa de Bradley e Wilk (2014) verificou que as mulheres negras eram mais propensas a 'parar de tra- 
balhar' durante as fases iniciais do tratamento. Ademais, nesse período, mais mulheres brancas encontravam-se empregadas e mais mulheres negras reportaram menor satisfação com o trabalho. Todavia, mais da metade das mulheres negras tinham mais probabilidade de estarem empregadas. As autoras postularam que, mesmo tendo de lidar com as consequências desfavoráveis do adoecimento, de modo geral as mulheres negras não podem abandonar o emprego devido aos encargos financeiros que assumem e, sobretudo, aqueles que impactam seu grupo familiar. Merece destaque que entre as mulheres inicialmente empregadas e que também dispunham de cobertura securitária, existiam poucas diferenças raciais em relação à $\mathrm{QV}$.

É válido comentar que o diagnóstico tardio das mulheres afro-americanas, mencionado por Bradley e Wilk (2014), converge com o que já foi observado no contexto brasileiro, aonde se percebe uma tendência em que mulheres negras recebem o diagnóstico de câncer de mama em estadiamento mais avançado (Nogueira et al., 2018; Renna Junior \& Silva, 2018). É preciso relembrar que quanto mais avançado for o estadiamento do câncer, maior será a agressividade do tratamento, o que poderá acarretar prejuízos para o exercício ocupacional (Paiva \& Cesse, 2015).

Morrow, Broxson, Munsell, Basen-Enquist, Rosenblum, Schover, Nguyen, Hsu, Castillo, Hahn, Litton, Kwiatkowski e Hortobaqyi (2014) mediram os efeitos a longo prazo do tratamento de câncer de mama em mulheres sobreviventes com idade igual ou menor a 45 anos e que haviam sido diagnosticadas há mais de um ano. Utilizaram, via correspondência, os instrumentos Quality of Life Cancer Survivors (QLACS), Breast Cancer Prevention Trial Symptom Checklist, Reproductive Concern Scale (RCS_ENREF_5) e um questionário demográfico. A amostra de 1.090 participantes teve $78 \%$ de respondentes brancas e cerca de $10 \%$ de mulheres negras e mulheres hispânicas, respectivamente. Morrow et al. (2014) ponderaram que aproximadamente um terço das participantes teve menopausa desencadeada pelo tratamento, mas somente $34 \%$ teve a chance de discutir sobre a possibilidade de infertilidade nesse contexto. As mulheres negras manifestaram mais distress relacionado a problemas financeiros do que mulheres brancas ou hispânicas, ao passo que estas últimas apresentaram um maior nível de preocupação associada à predisposição familiar ao câncer de mama e uma pontuação pior nas escalas específicas de câncer. $\mathrm{Na}$ esfera da sexualidade, pacientes diagnosticadas há menos de cinco anos reportaram mais queixas de secura vaginal e dor na relação sexual quando comparadas com aquelas que tinham mais de 10 anos. As pacientes com mais de 40 anos comunicaram menor interesse sexual.

Em 2015, Gaston-Johansson, Watkins, Kanu, Whitehouse, Sarenmalm, Brovall e Kozachik publicaram uma investigação de natureza descritiva e longitudinal com 30 mulheres afro-americanas. A amostra foi avaliada desde o início do tratamento até uma semana após a conclusão da quimioterapia com aplicação de: The Pain-O-Meter (POM); Visual Analog Scale (VAS) para medida de fadiga e náusea; Hospital Anxiety and Depression Scale (HADS); Insomnia Severity Index (ISI) e Functional Assessment of Cancer Therapy Breast (FACT-B) e um questionário sociodemográfico. Por ocasião da etapa intermediária da quimioterapia e ao final desse tratamento, todos os sintomas intensificaram-se. Tanto a dor como a depressão tiveram efeitos negativos significativos sobre a QV. A pesquisa estimou ainda que mais de $70 \%$ da amostra não havia adotado deliberadamente estratégias de enfrentamento (por exemplo: relaxamento) no último ano. A maioria afirmou não ter recebido assistência psicológica e psiquiátrica nos últimos cinco anos. Ao longo do tempo, os sintomas depressivos mostraram-se compatíveis com níveis próximos ao patológico. A ansiedade se manteve constante ao longo do tratamento, porém 10 participantes reportaram ansiedade anormal já na linha de base da avaliação.

Samuel, Pinheiro, Reeder-Hayes, Walker, Corbie-Smith, Fashaw, Woods-Giscombe e Wheeler (2016) empreenderam uma revisão sistemática de 1995 a 2015 nas bases MEDLINE/PubMed, EMBASE, CINAHL e PsycINFO, tendo encontrado, inicialmente, 2.533 artigos. Porém, apenas seis artigos preencheram os critérios definidos pelas autoras. O levantamento mostrou que, ao serem comparadas com mulheres mais velhas, brancas e sem experiência anterior com essa doença, as sobreviventes negras relataram mais medo de morrer, necessidades de cuidados não atendidas, dificuldades financeiras e menor bem-estar físico/funcional. A dimensão bem-estar espiritual revelou-se mais favorável para as sobreviventes negras.

Pinheiro, Samuel, Reeder-Hayes, Wheeler, Olshan e Reeve (2016) analisaram diferenças raciais da QV durante a etapa de tratamento ( $5^{\circ}$ mês) e a etapa de sobrevivência ( $25^{\circ}$ mês) ao câncer de mama. Para isso, usaram dados de um estudo realizado na Carolina do Norte (Estados Unidos) com mulheres entre 20 e 74 anos, os quais foram coletados mediante a aplicação de The Functional Assessment of Cancer Therapy for Breast Cancer (FACT-B) e Functional Assessment of Chronic Illness Therapy for Spirtual Well-Being (FACIT-SP). Participaram 2.142 mulheres brancas $(n=$ 


\section{ESTHÉR APARECIDA SILVA DOS SANTOS \\ TEREZA CRISTINA CAVALCANTI FERREIRA DE ARAUJO}

1.105) e negras $(n=1.037)$. Nas duas etapas, registraram-se escores físicos e funcionais mais desfavoráveis entre as mulheres negras. Quanto aos aspectos espirituais, os escores alcançados foram melhores nestas últimas. Essa evidência alerta para a importância de um manejo clínico adequado por parte da equipe de saúde em relação ao enfrentamento religioso/espiritual, a depender da dimensão étnica da paciente. É crucial salientar que a literatura admite modalidades mais ou menos adaptativas desse tipo de enfrentamento (Gobatto \& Araujo, 2013).

Com preocupações semelhantes, a publicação de Pinheiro, Tan, Olshan, Wheeler, Reeder-Hayes, Samuel e Reeve (2017) também se apoiou nos dados do estudo executado na Carolina do Norte e usou os resultados gerados pelos instrumentos supracitados e coletados nos meses previamente mencionados. Desta feita, os autores distinguiram quatro perfis latentes (PL) de QV, de PL1 (pior) até PL4 (melhor). No $5^{\circ}$ mês, fatores associados a um perfil inferior foram: ser mulher branca, jovem, não casada, ter comorbidade, ser tabagista, passar por tratamento mais intenso e não ter plano de saúde. No $25^{\circ}$ mês, destacaram-se: ser mulher negra, ter menos idade, comorbidades, tabagismo e obesidade. Essa pesquisa reforçou que as condições de vida da mulher negra podem impactar negativamente a QV.

Vadaparampil, Christie, Donovan, Kim, Augusto, Kasting, Holt, Ashing, Halbert e Pal (2017) compararam mulheres negras com câncer denominado 'triplo-negativo' e mulheres não portadoras desse tipo histológico de câncer, empregando vários instrumentos e mensurando a QV com auxílio da Functional Assessment of Cancer Therapy - Breast (FACT-B). Averiguaram que as participantes do primeiro grupo possuíam uma pior percepção de QV. As mulheres negras jovens, com diagnóstico de 'triplo-negativo', informaram mais perdas de QV. Independentemente do tipo de câncer, fatores individuais - como autopercepção ruim da própria saúde, maior limitação funcional, estar em quimioterapia e alto nível de ansiedade - estavam relacionados à insatisfação com a QV. Cumpre enfatizar que o tipo triplo-negativo corresponde a um tumor mais agressivo, sendo que a sua taxa de incidência tende a ser maior em mulheres mais jovens e de ascendência africana ou hispânica (Corrêa, Toralles, Abe-Sandes, Machado \& Bonfim, 2010; Jiagge, Chitale, \& Newman, 2018). Ao se refletir sobre a associação entre diagnóstico tardio e uma maior propensão para desenvolver um câncer de mama que exigirá mais cuidados, confirma-se a relevância de mais investigações para fundamentar intervenções de ordem preventiva e terapêutica voltadas para esse segmento da população.

Mais recentemente, Claridy, Ansa, Damus, Alema-Mensah e Smith (2018) focalizaram apenas a QV de afro-americanas, as quais foram distribuídas em três subamostras: sobreviventes de câncer de mama, sobreviventes de outros tipos de câncer e sem histórico da doença. A pesquisa não detectou diferenças significativas entre sobreviventes de câncer de mama e mulheres que não tiveram câncer. De modo geral, as sobreviventes de outros tipos de câncer relataram pior saúde física e mental do que mulheres sem histórico de câncer. Sobreviventes que tinham seguro privado relataram melhor saúde física e mental do que aquelas que não tinham seguro privado. Sobreviventes de câncer de mama relataram saúde física e mental um pouco melhor do que sobreviventes de outros tipos de câncer. A resiliência dessas sobreviventes oncológicas pode ser melhor compreendida quando se presume que o estigma relacionado a esse tipo de câncer diminuiu devido ao seu maior destaque nas mídias e à melhoria dos indicadores de cura. Pressupõe-se, igualmente, que essas mulheres contaram com fontes de suporte social.

Conforme explicitado no presente artigo, o constructo QV caracteriza-se pela complexidade e multideterminação. Diante disso, pode-se evidenciar, nos oito trabalhos anteriormente reportados, que o contexto socioeconômico é um aspecto relevante para compreensão da QV de mulheres negras com câncer de mama, notadamente no que concerne o emprego e o acesso aos serviços especializados mediante planos de saúde, em cenários internacionais.

Nos trabalhos de Samuel et al. (2016) e Claridy, Ansa, Damus, Alema-Mensah e Smith (2018), o fator 'idade' das pacientes igualmente se destacou. No caso de Samuel et al. (2016), ao se considerar a idade (menos de 50 anos) como um fator de influência na percepção de QV de mulheres negras com câncer, questões como o desejo da maternidade e outras decorrentes das vivências de uma fase de vida - geralmente marcada por mais expectativas em diferentes domínios da existência, podem estar relacionadas a uma percepção mais negativa da QV nessa faixa etária. É importante reprisar que Morrow et al. (2014) abordaram o efeito adverso 'infertilidade', sendo que muitas participantes revelaram que não se lembravam de terem discutido sua saúde reprodutiva, a despeito do desejo da maternidade por ocasião do diagnóstico e início do tratamento. Essas reflexões remetem a limitações persistentes na relação paciente-profissional de saúde. Isso se hipertrofia quando se sobrepõe a discriminação racial, tal como discutido por Domingues, Nascimento, 
Oliveira, Barral, Rodrigues, Santos e Araújo (2013). Ou seja, possíveis tensões raciais podem ser demonstradas por atitudes enviesadas por parte do profissional, restringindo uma participação mais ativa da paciente no sentido de atender às suas necessidades. Mulheres negras podem se sentir desconfortáveis ao questionarem orientações e expressarem dúvidas, o que pode perpetuar crenças distorcidas.

Nos trabalhos enfocados no levantamento aqui exposto, enfrentamento religioso/espiritual surgiu como uma modalidade estratégica adotada por mulheres negras, a exemplo de outros segmentos populacionais. De modo semelhante, Yoo, Levine e Pasick (2014) perceberam que mulheres negras acometidas por um câncer de mama, tendiam a utilizar mais recursos de espiritualidade e religiosidade como estratégia de enfrentamento.

Cabe frisar que os artigos aqui examinados mostraram que a incidência de sintomas depressivos e ansiosos é fator desfavorável para a QV de mulheres negras, o que confirma a necessidade de institucionalização de protocolos clínicos, devidamente baseados em pesquisas, para contemplar padrões desejáveis de qualidade da assistência em Oncologia (Bergerot \& Araujo, 2017; Muniz, Zago, \& Schwartz, 2009).

Com relação à sobrevivência, é imprescindível assinalar que Samuel et al. (2016), Pinheiro et al. (2016), Pinheiro et al. (2017), Vadaparampil et al. (2017) e Claridy, Ansa, Damus, Alema-Mensah e Smith (2018) não concordaram quanto aos parâmetros temporais referentes a essa etapa da experiência oncológica. Seus estudos acataram desde o período de recebimento do diagnóstico até um período indeterminado que pode ser confundido com o próprio ciclo vital da paciente. De fato, existe uma variedade de definições de sobrevivência na literatura especializada e alguns autores contestam a adoção de critérios rígidos. Mas, compete atentar que o uso de períodos temporais discrepantes limita as possibilidades de estudos de meta-análise sobre o assunto (Naves \& Araujo, 2015).

Por fim, é relevante mencionar que sociedades representativas da Oncologia (American Society of Clinical Oncology \& European Society for Medical Oncology, 2006) sublinham que é essencial assegurar que elementos de discriminação não interfiram no atendimento. Entretanto, parecem perdurar fatores - como dificuldade de acesso aos meios preventivos e terapêuticos, informações deficitárias e vieses na relação profissional e paciente -, os quais prejudicam, sinergicamente, uma assistência integral e de qualidade.

\section{Considerações Finais}

Em suma, a revisão da literatura desenvolvida no presente artigo indica que sintomas depressivos e ansiosos, faixa etária inferior a 50 anos, quimioterapia e diagnóstico de triplo negativo estão associados a uma percepção de QV mais desfavorável entre mulheres negras com câncer de mama. Em compensação, estratégias adaptativas de ordem religiosa/espiritual vinculam-se a uma percepção mais positiva.

É notória a premência por ações em favor do empoderamento da mulher negra. Na esfera da saúde e, em especial em Oncologia, isso deve ser priorizado, uma vez que a literatura já revela especificidades que devem nortear programas e políticas na área.

Conclui-se que são necessários mais estudos para explorar variáveis étnicas e sociais, pois os cuidados de saúde ocorrem em contextos interacionais, nos quais tais aspectos influenciam as relações usuário-profissional; usuário-equipe e usuário-instituição. Particularmente no campo da Psico-Oncologia, é imprescindível desenvolver investigações sobre QV, enfrentamento, distress e resiliência que enfoquem especificidades étnico-culturais. Sugerem-se estudos multicêntricos, preferencialmente longitudinais, com amostras de espectro nacional e regional.

\section{Referências bibliográficas}

American Society of Clinical Oncology, \& European Society for Medical Oncology. (2006). Consensus statement on quality cancer care. Annals of Oncology, 17(7), 1063-1064.

Bergerot, C. D., \& Araujo, T. C. C. F. (2017). Desenvolvimento e aplicação de protocolos clínicos em Psico-Oncologia: Perspectivas para atuação em equipe de saúde especializada. In D. M. Amparo, E. R. Lazzarini, I. M. Silva \& L. Polejack. (Orgs.), Psicologia Clínica e Cultura Contemporânea 3 (pp. 583-605). Brasília: Technopolitik.

Bergerot, C. D., Araujo, T. C. C. F. \& Tróccoli, B. T. (2014). Assessment of distress among chemotherapy patients: A comparative study of gender. Paidéia, 24, 57, 56-65.

Bradley, C. J., \& Wilk, A. (2014). Racial differences in quality of life and employment outcomes in insured women with breast cancer. Journal of Cancer Survivorship, 8, 49-59.

Claridy, M. D., Ansa, B., Damus, F., Alema-Mensah, E., \& Smith, S. A. (2018). Health-related quality of life of African-American female breast cancer survivors, survivors of other cancers, and those without cancer. Quality of Life Research, 27(8), 2067-2075.

Corrêa, P. B., Toralles, M. B. P., Abe-Sandes, K., Machado, T. M. B., \& Bonfim, T. M. (2010). Câncer de mama triplo-negativo e sua associação com ancestralidade africana. Revista de Ciências Médicas e Biológicas, 9(1), 3-7.

Costa Neto, S. B., \& Araujo, T. C. C. F. (2008). Qualidade de vida em enfermo oncológico: Um panorama sobre o campo e suas formas de avaliação. In V. A. Carvalho, M. H. P. Franco, M. J. Kovács, R. P. Liberato, R. C. Macieira, M. T. Veit, M. J. B. Gomes, \& L. H. C. Barros (Orgs.), Temas em Psico-oncologia (pp. 195-208). São Paulo: Summus.

Domingues, P. M. L., Nascimento, E. R., Oliveira, J. F., Barral, F. E., Rodrigues, Q. P, Santos, C. C. C., \& Araújo, E. M. (2013). Discriminação racial no cuidado em saúde reprodutiva na percepção de mulheres. Texto \& Contexto - Enfermagem, 22(2), 285-292.

Fleck, M. P. A. (Org.) (2008). A avaliação de qualidade de vida: Guia para profissionais da saúde. Porto Alegre: Artmed. 


\section{ESTHÉR APARECIDA SILVA DOS SANTOS \\ TEREZA CRISTINA CAVALCANTI FERREIRA DE ARAUJO}

Galvão, T. F., \& Pereira, M. G. (2014). Revisões sistemáticas da literatura: Passos para sua elaboração. Epidemiologia e Serviços de Saúde, 23(1), 183-184.

Gaston-Johansson, F., Watkins, C. C., Kanu, I. K., Whitehouse, E., Sarenmalm, E. K., Brovall, M., \& Kozachik, S. L. (2015). The effects of symptoms on quality of life during chemotherapy in african-american women with breast cancer. Journal of the National Black Nurses Association, 26(2), 7-16.

Gobatto, C. A., \& Araujo, T. C. C. F. (2013). Religiosidade e espiritualidade em oncologia: Concepções de profissionais da saúde. Psicologia USP, 24, 11-34.

Gurung, R. A. R. (2010). Health psychology. A cultural approach. Wadsworth: Cengage Learning.

Instituto Nacional de Câncer José Alencar Gomes da Silva. (2018). A mulher e o cancer de mama no Brasil ( $3^{a}$ ed.). Rio de Janeiro: INCA.

Instituto Nacional de Câncer José Alencar Gomes da Silva. (2019). A situação do câncer de mama no Brasil: Síntese de dados do sistema de informação. Rio de Janeiro: INCA.

Jiagge, E., Chitale, D., \& Newman, L. A. (2018). Triple-negative breast cancer, stem cells, and african ancestry. The American Journal of Pathology, 188(2), 271-279.

López, L. C. (2012). O conceito de racismo institucional: Ampliações no campo da saúde. Interface - Comunicação, Saúde, Educação, 16(40), 121-134.

Matos, D. R., \& Araujo, T. C. C. F. (2015). Avaliação em reabilitação: Perspectivas para qualidade de vida (pp. 155-180). In T. C. C. F. Araujo, \& E Queiroz. (Orgs.), Psicologia da Reabilitação. Perspectivas teóricas, metodológicas e práticas. Brasília: Liber Livro.

Ministério da Saúde. (2013). Política nacional de saúde integral da população negra: Uma politica do SUS (2a ed.). Brasília: MS.

Morrow P. K., Broxson, A. C., Munsell, M. F., Basen-Enquist, K., Rosenblum, C. K., Schover, L. R., Nguyen, L. H., Hsu, L., Castillo, L., Hahn, K. M., Litton, J. K., Kwiatkowski, D. N. \& Hortobagyi, G. N. (2014). Effect of age and race on quality of life in young breast cancer survivors. Clinical Breast Cancer, 14(2), e21-31.

Muniz, R. M., Zago, M. M. F., \& Schwartz, E. (2009). As teias da sobrevivência oncológica: Com a vida de novo. Texto \& Contexto - Enfermagem, 18(1), 25-32.

Naves, J. F., \& Araujo, T. C. C. F. (2015). Qualidade de vida e bem-estar subjetivo de sobreviventes ao câncer ósseo: Percepção de sobreviventes e familiares. Interação em Psicologia, 19(3), 351-363.

Nogueira, M. C., Guerra, M. R., Cintra, J. R. D., Corrêa, C. S. L., Fayer, V. A., \& Bustamante-Teixeira, M. T. (2018). Disparidade racial na sobrevivência em 10 anos para o câncer de mama: Uma análise de mediação usando abordagem de respostas potenciais. Cadernos de Saúde Pública, 34(9), 1-15.

Paiva, C. J. K., \& Cesse, E. A. P. (2015). Aspectos relacionados ao atraso no diagnóstico e tratamento do câncer de mama em uma unidade hospitalar de Pernambuco. Revista Brasileira de Cancerologia, 61(1), 23-30.
Pinheiro, L. C., Samuel, C. A., Reeder-Hayes, K. E., Wheeler, S. B., Olshan, A. F., \& Reeve, B. B. (2016). Understanding racial differences in health-related quality of life in a population-based cohort of breast cancer survivors. Breast Cancer Research and Treatment, 159(3), 535-543.

Pinheiro, L. C., Tan, X., Olshan, A. F., Wheeler, S. B., Reeder-Hayes, K. E., Samuel, C. A., \& Reeve, B. B. (2017). Examining health-related quality of life patterns in women with breast cancer. Quality of Life Research, 26(7), 1733-1743.

Renna Junior, N. L., \& Silva, G. A. (2018). Tendências temporais e fatores associados ao diagnóstico em estágio avançado de câncer do colo uterino: Análise dos dados dos registros hospitalares de câncer no Brasil, 2000-2012. Epidemiologia e Serviços de Saúde, 27(2), 1-13.

Samuel, C. A., Pinheiro, L. C., Reeder-Hayes, K. E., Walker, J. S., Corbie-Smith, G., Fashaw, S. A., Woods-Giscombe, C., \& Wheeler, S. B. (2016). To be young, Black, and living with breast cancer: A systematic review of health-related quality of life in young Black breast cancer survivors. Breast Cancer Research and Treatment, 160(1), 1-15.

Silva, D. W. R., Araujo, H. A., \& Câmara, J. T. (2017). Mortalidade por câncer de mama entre mulheres negras e brancas: Uma revisão integrativa. In Anais da VI Jornada multidisciplinar de Biologia e Saúde e IX Mostra Cientifica do CESC-UEMA. Caxias: UEMA.

Vadaparampil, S. T., Christie, J., Donovan, K. A., Kim, J., Augusto, B., Kasting, M. L., Holt, C. L., Ashing, K., Halbert, C. H., \& Pal, T. (2017). Health-related quality of life in Black breast cancer survivors with and without triple-negative breast cancer (TNBC). Breast Cancer Research and Treatment, 163(2), 331-342.

Vieira, R. A. C., Formenton, A., \& Bertolini, S. R. (2017). Breast cancer screening in Brazil. Barriers related to the health system. Revista da Associação Médica Brasileira, 63(5), 466-474.

World Health Organization. (2018). Latest global cancer data: Cancer burden rises to 18.1 million new cases and 9.6 million cancer deaths in 2018. Disponível em https://www.iarc.fr/wp-content/uploads/2018/09/pr263 E.pdf

Yin, S., Njai, R., Barker, L., Siegel, P. Z., \& Liao, Y. (2016). Summarizing health-related quality of life (HRQOL): Development and testing of a one-factor model. Population Health Metrics, 14, 22.

Yoo, G. J., Levine, E. G., \& Pasick, R. (2014). Breast cancer and coping among women of color: A systematic review of the literature. Supportive Care in Cancer, 22(3), 811-824.

Submetido em: 1-5-2020

Aceito em: 3-1-2021 